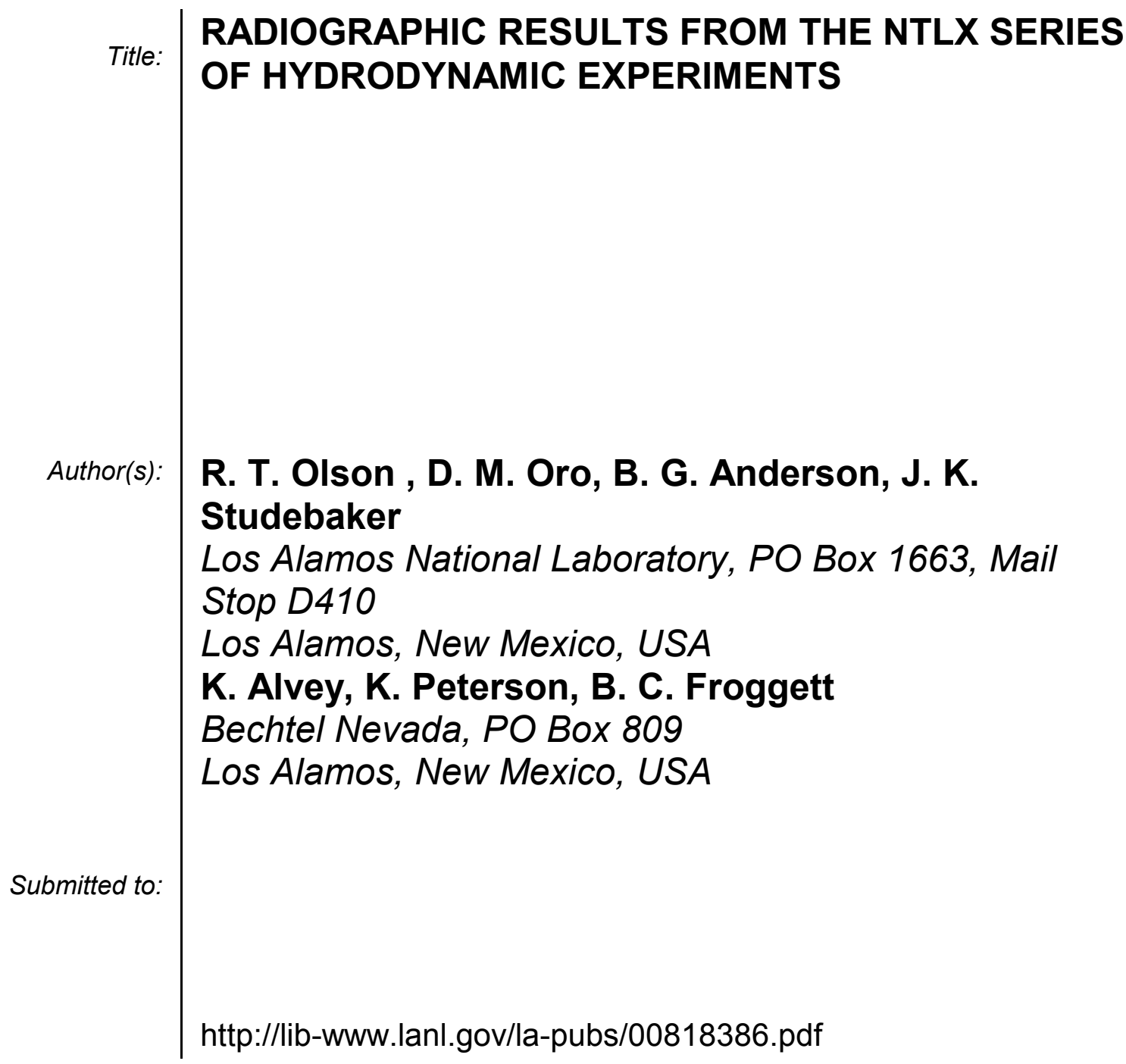




\title{
RADIOGRAPHIC RESULTS FROM THE NTLX SERIES OF HYDRODYNAMIC EXPERIMENTS*
}

\author{
R. T. Olson ${ }^{\xi}$, D. M. Oro, B. G. Anderson, J. K. Studebaker \\ Los Alamos National Laboratory, PO Box 1663, Mail Stop D410 \\ Los Alamos, New Mexico, USA
}

\author{
K. Alvey, K. Peterson, B. C. Froggett \\ Bechtel Nevada, PO Box 809 \\ Los Alamos, New Mexico, USA
}

\begin{abstract}
The NTLX series of experiments are focused on measuring the shock induced hydrodynamic flow of a SnPMMA target. For these experiments multi-frame flash $\mathrm{X}$-ray radiography is used to measure the position of the Sn-PMMA target interface and the location of shock in the PMMA as a function of time. Four radiographs are acquired at $700 \mathrm{~ns}$ intervals having a line-of-sight following the target's axis of symmetry. Because the Xray spectrum from the sources has an end-point energy of $\sim 300 \mathrm{keV}$ with a strong component of $\sim 60 \mathrm{keV}$ tungsten $\mathrm{K}$-line radiation, the $\mathrm{Sn}$ portion of the target is radiographically opaque. However, X-rays are transmitted through the PMMA portion of the target thereby allowing motion of the Sn-PMMA interface to be imaged. Also, the shock location is tracked as a function of time due to the density increase in the shocked PMMA.

The resulting radiographs are analyzed to provide the trajectory and shape of both the shock and Sn-PMMA interface. In addition, the shock velocity in the $\mathrm{Sn}$ is determined for asymmetric target geometries.
\end{abstract}

\section{I.INTRODUCTION}

The NTLX (Near Term Liner eXperiments) series of experiments are designed to provide benchmark data for various hydrodynamic codes currently used to predict the behavior of shocked materials. These experiments were performed using the Shiva Star capacitor bank to magnetically implode an Al liner onto a Sn-PMMA (tinPolyMethyl MethAcrylate commonly known as Lucite®) target [1]. The liner impact launched an $\sim 1$ Mbar converging cylindrical shock into either a symmetric target design used in NTLX-1 and NTLX-3 or an asymmetric target used in NTLX-2 and NTLX-4. The symmetric target consists of a $40 \mathrm{~mm}$ diameter Sn cylinder with a coaxial PMMA core $30 \mathrm{~mm}$ in diameter. The asymmetric target has the same dimensions except the PMMA axis is offset $4 \mathrm{~mm}$ from the target axis as defined by the outer diameter of the Sn. The Al liner impacts the outer surface of the Sn symmetrically with nominally the same drive conditions for each experiment in the NTLX series.

The trajectories and velocities of both the shock and material interfaces in the target are of particular interest for hydrodynamic code validation. As a result, a multiframe X-radiographic technique was used to axially image the shock induced hydrodynamic flow in the targets as a function of time. This article briefly describes the X-radiographic diagnostic fielded for the NTLX series and presents both the acquired images and the data extracted from them.

\section{II.X-RADIOGRAPHIC TECHNIQUE}

Four vertically stacked tungsten anode X-ray diode sources are fired independently to create four temporally resolved radiographs. Each source is driven with a Los Alamos National Laboratory designed 900 keV Marx bank that delivers an $\sim 20$ ns wide X-ray pulse [2]. The

\footnotetext{
* Work supported by the Los Alamos National Laboratory under US DOE contract W-7405-ENG-36

६ email: rtolson@lanl.gov
} 


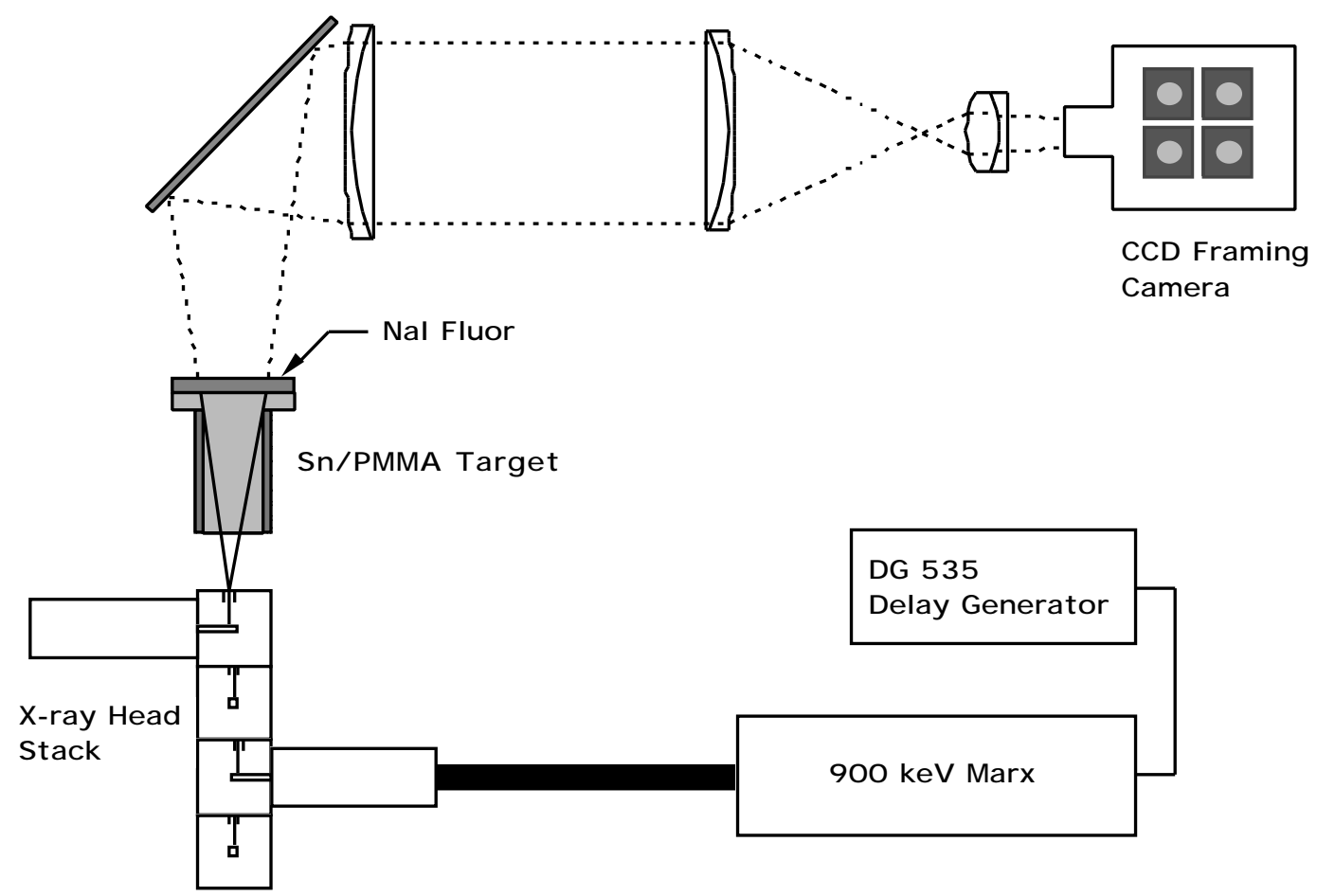

Figure 1. Schematic illustrating the axial X-radiography diagnostic fielded for the NTLX experimental series.

source spectrum is composed of broad band Bremstrahlung radiation with an endpoint energy of $\sim 300$ $\mathrm{keV}$ combined with a strong component of tungsten $\mathrm{K}$ line radiation.

The X-ray source stack is rigidly held below the target and is aligned with the PMMA symmetry axis in the target. A $1.0 \mathrm{~mm}$ thick $\mathrm{NaI}$ fluor with an exponential decay time of $230 \mathrm{~ns}$ rests on top of the target and is used as the X-ray to light converter. The resulting geometric magnification of the target mid plane onto the fluor ranges from 1.09 to 1.07 .

The image on the fluor is transported to a 16 bit, $1024 \times 1024$ CCD camera via the optical transport system shown schematically in Figure 1. A turning mirror, two 8 inch diameter doublet lenses, a 4 inch diameter field lens, and a commercial $50 \mathrm{~mm}$ Olympus macro lens create a $f 2.7$ optical system with a total path length of $\sim 5 \mathrm{~m}$. The fluor is imaged onto an MCP (Micro Channel Plate) image intensifier that is coupled to an electronic framing tube in front of the CCD. The framing tube allows the image generated on the fluor by each X-ray source to be imaged on a separate quadrant of the CCD.

The X-radiograph acquisition times are set to coincide with the shock propagation through the PMMA portion of the target using pre-shot as a guide. The time each X-ray source fires is measured using a PIN photodiode and defines the image acquisition time. A minimum of $700 \mathrm{~ns}$ (3 fluor decay times) is required between each X-ray pulse to prevent a bright latent image on the fluor from being superimposed with subsequent radiographs.

The Marx banks that drive the X-ray sources are fired via a delay generator triggered by the Shiva Star command trigger and have an $\sim 20$ ns shot-to-shot jitter. However, because the time each X-ray source fires is individually monitored during the experiment, the dominate timing uncertainty is caused by motional blur associated with the finite $\mathrm{X}$-ray source pulse length.

\section{III.NTLX RADIOGRAPHIC ANALYSIS}

The X-radiographic data obtained during each NTLX experiment consists of X-ray source firing times obtained from the PIN photodiodes, a static image of the target acquired prior to the experiment, and the dynamic image taken during shock propagation through the target. The dynamic radiographic data obtained from the asymmetric target used during NTLX-4 are shown in Figure 2. To extract quantitative shock and Sn-PMMA interface data from the four temporally resolved X-radiographs, each image must undergo multiple processing steps. 

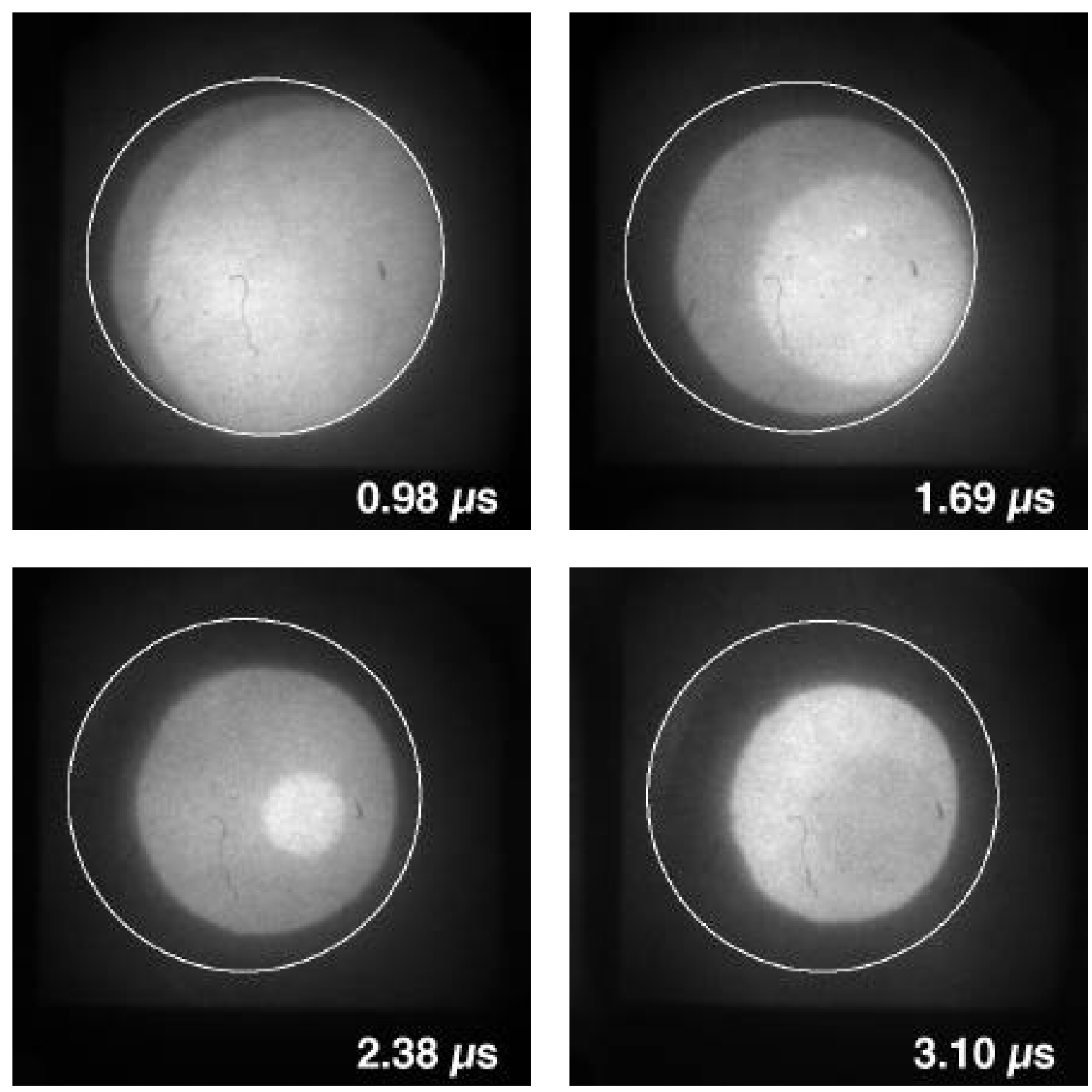

Figure 2. The NTLX-4 dynamic X-radiographs showing the hydrodynamic flow of the asymmetric Sn-PMMA target. The superimposed circle indicates the Sn-PMMA interface location extracted from the static Xradiographs. Each image shows both the shock propagation through the PMMA and the motion of the SnPMMA interface. The X-radiograph acquisition times are measured from liner impact on the target.

Initially, the image is background subtracted and each of the four $\mathrm{X}$-radiographs recorded in the single $1024 \times 1024$ pixel image is extracted as an individual $512 \times 512$ pixel image. Each extracted X-radiographic static and dynamic frame contains image distortions introduced by the both optical transport system and electronic framing tube in the camera. A 2-dimensional, $5^{\text {th }}$ order polynomial is generated from the image of a reference object and used to minimize these distortions using a digital image warp procedure. Finally, the static $\mathrm{X}$-radiograph is registered to the dynamic to correct for slight deviations in image placement by the electronic framing tube.

There are three features of interest in each NTLX radiograph: shock location in the PMMA and the SnPMMA interface location in both the static and dynamic. Background image noise is reduced using a median filter operation designed to preserve the edge locations and 
shapes. Further noise reduction and edge enhancement is achieved using a line weight function (LWF) filter. The LWF is modeled on the contrast sensitivity of the human eye and is a circularly symmetric combination of a Gaussian and its second derivative [3]. The peak gradient across each edge in the radiographs is defined as the edge location that is found using a Sobel derivative filter.

Shock and Sn-PMMA interface data are extracted from the resulting filtered, Sobel differentiated image. This data consists of pairs of $x$ - and $y$-pixel values that define each edge of interest. Assuming the Al liner impacts uniformly on the outside of the target, the shock generated within the PMMA should be circular for the symmetric targets and elliptical for the asymmetric targets. Therefore, a geometric least squares fit of either a circle or an ellipse is applied as appropriate to the extracted edge data points. These best fit edge results yield information on convergence point, trajectory via the radius or major and minor axis, ellipse rotation angle, and the error in determining the edge position.

The edge data is scaled from pixel to target dimensions using the known $30 \mathrm{~mm}$ diameter of the PMMA. The scaling is uniquely determined for each dynamic frame from its associated static because of the different geometric X-ray magnifications. In addition, the vertical location of the shock and Sn-PMMA interface within the target that creates the X-ray opacity must be identified to properly account for X-ray magnification differences.

\section{IV.X-RADIOGRAPHIC RESULTS}

The X-radiographic measurements of shock position in the PMMA are shown in Figure 3 with a calculated error of less than $\pm 0.15 \mathrm{~mm}$. Performing a linear least squares fit to all the data points gives an average shock speed in the PMMA of $8.0 \mathrm{~mm} / \mu \mathrm{s}$. Only the data point acquired later in time during NTLX-1 shows any indication of shock acceleration due to the convergent experimental geometry. The Sn-PMMA interface clearly shows a deceleration of the Sn as the PMMA is compressed (See Figure 3). However, no radiographic data were collected late enough in time to observe the maximum compression point or subsequent expansion.

The extracted shock and Sn-PMMA edges from the asymmetric targets were fit with ellipses to quantify the shape. However, no significant ellipticity was detected that could not be explained simply by the $\pm 0.15 \mathrm{~mm}$ uncertainty in edge location. This is an unexpected result because calculations indicate the differnces in sound

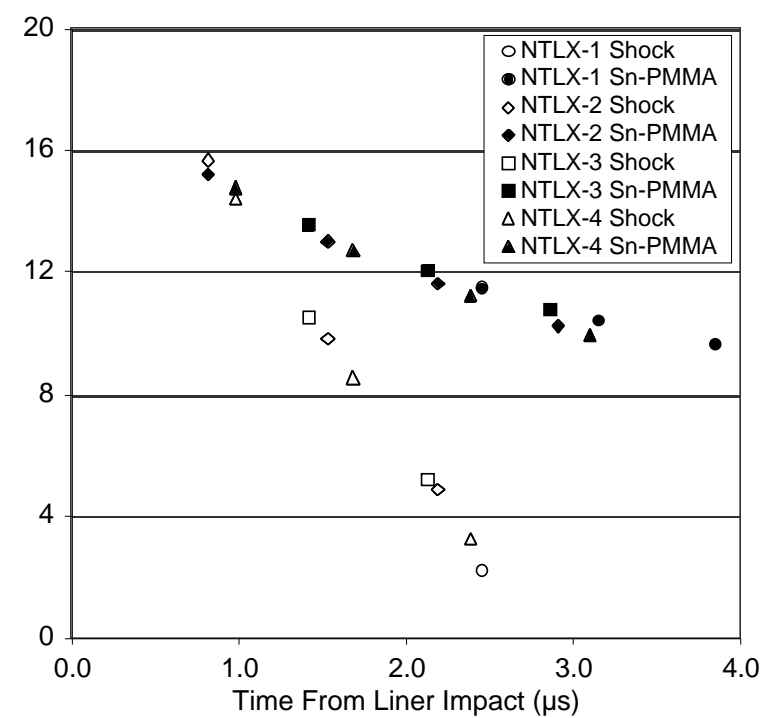

Figure 3. Plot of shock and Sn-PMMA radius vs. time measured from liner impact on the target.

speed between the Sn and PMMA should create an ellipticity of $\sim 15 \%$.

Although the $\mathrm{Sn}$ portion of the target is radiographically opaque, it is possible to determine average shock velocity through the Sn for the asymmetric target design using the $\mathrm{X}$-radiographic data. This is achieved using the images acquired where the shock has only partially entered the PMMA from the Sn. A calculation of the intercept point between the best fit static Sn-PMMA edge and the best fit shock boundary provides the shock breakout point at the $\mathrm{X}$-radiograph acquisition time. The average shock velocity in the $\mathrm{Sn}$ is then calculated using the liner impact time and $\mathrm{Sn}$ thickness at the breakout point. This technique provides a $\mathrm{Sn}$ shock velocity of $5.76 \mathrm{~mm} / \mu \mathrm{s}$ and $5.81 \mathrm{~mm} / \mu$ for NTLX-2 and NTLX-4, respectively.

Additional information relating to the NTLX series can be found in companion articles published elsewhere in these proceedings.

\section{V.REFERENCES}

[1] J. H. Degnan, et al., "Multi-Megajoule Solid Liner Implosions", in Mega Gauss Technology and Pulsed Power Applications, 1986, p. 699.

[2] D. Platts, et al., "Compact Flash X-ray Units", in $10^{\text {th }}$ IEEE International Pulsed Power Conference, 1995, p. 892.

[3] L. M. Kennedy and M. Basu, "Image enhancement using a human visual system model", Pattern Recognition, vol. 30, pp. 2001-2014, (1997). 